\title{
Uma metodologia de aprendizagem vivencial para o desenvolvimento de competências para o gerenciamento da implementação de sistemas de informação
}

\author{
Alexandre Cidral (UNIVILLE) cidral.joi@terra.com.br \\ Avanilde Kemczinski (UDESC) avanilde@terra.com.br \\ Aline França de Abreu (UFSC) aline@eps.ufsc.br
}

\section{Resumo}

Este artigo apresenta a fundamentação teórica e o delineamento de uma metodologia de aprendizagem vivencial para o desenvolvimento de competências para o gerenciamento da implementação de sistemas de informação. A metodologia de aprendizagem vivencial está em fase inicial de aplicação em cursos superiores da área de Computação e Informática e da área de Engenharia de Produção.

Palavras chave: Metodologia de ensino, Aprendizagem vivencial, Implementação de sistemas de informação.

\section{A implementação de sistemas de informação}

Um sistema de informação é um conjunto de componentes inter-relacionados para recuperação, processamento, armazenamento e distribuição da informação, que oferece suporte à tomada de decisão, à coordenação e ao controle organizacionais, à análise de situações complexas e à criação de novos produtos e serviços (LAUDON e LAUDON, 1999). Entretanto, a efetividade dos sistemas de informação só é alcançada a partir de um processo de implementação que seja capaz de integrar as dimensões tecnológica, humana e organizacional destes sistemas. Assim, a implementação de sistemas de informação:

- é um processo (GINZBERG, 1979) realizado ao longo de todo o ciclo de vida do sistema (LUCAS, 1981);

- busca a melhoria do desempenho organizacional (LAUDON e LAUDON, 1999);

- caracteriza-se como processo de mudança nas dimensões organizacional, humana e tecnológica ( LUCAS, GINZBERG e SCHULTZ, 1990), na medida que exige um ajuste mútuo entre organização, pessoas e tecnologia (BIKSON e EVELAND, 1990);

- é uma inovação tecnológica (TORNATZKY e FLEISCHER, 1990) (KWON e ZMUD, 1987) que abrange adoção, gerenciamento e rotinização de um sistema de informação;

- é um projeto (PINTO e MILLET, 1999) que exige o gerenciamento através de metodologias, técnicas e ferramentas apropriadas;

- é um processo de aprendizagem (APPLEGATE et al, 1999) empreendido por uma equipe que deve desenvolver competências específicas para atuar na implementação e posterior utilização e gerenciamento do sistema.

Apesar da importância dos sistemas de informação, nem sempre a sua implementação alcança os objetivos a que se propõe, fazendo com que o sucesso/fracasso destes projetos constitua um tema de pesquisa em sistemas de informação. Há um certo consenso na classificação das pesquisas em estudos sobre fatores e estudos sobre processos (LUCAS, GINZBERG e SCHULTZ, 1990). Além disso, os pesquisadores indicam a necessidade de construir modelos 
capazes de integrar as contribuições provenientes destes dois focos (LUCAS, GINZBERG e SCHULTZ, 1990). Esta integração propiciaria uma melhor compreensão do tema e a operacionalização de intervenções mais efetivas sobre os problemas relacionados à implementação. Considera-se que o principal foco de problemas diz respeito a questões organizacionais e humanas, na medida que estes aspectos exercem maior influência sobre o processo de mudança inerente à implementação.

No que diz respeito aos estudos sobre o processo de implementação, o modelo de desenvolvimento organizacional (Quadro 1) (KOLB e FROHMAN, 1970), tornou-se útil ao abordar a relação entre o profisssional de sistemas de informação (consultor) e os usuários do sistema (clientes) ao longo das etapas da implementação.

\begin{tabular}{|l|l|}
\hline Estágio & Atividades \\
\hline Exploração & $\begin{array}{l}\text { Cliente e consultor determinam as necessidades e capacidades um do outro. A área da } \\
\text { organização que receberá o novo sistema é avaliada. }\end{array}$ \\
\hline Entrada & $\begin{array}{l}\text { A declaração inicial de objetivos do sistema é realizada. Busca-se o comprometimento e } \\
\text { confiança entre os instaladores do sistema e o s usuários. Cria-se uma necessidade de mudança. }\end{array}$ \\
\hline Diagnóstico & Dados são coletados para determinar o que especificamente os clientes buscam. \\
\hline Planejamento & Os objetivos específicos, marcos de referência, atividades e recursos são definidos. \\
\hline Ação & $\begin{array}{l}\text { O sistema é colocado em funcionamento. As modificações necessárias no plano ou no sistema } \\
\text { são feitas em resposta a eventos e contingências. }\end{array}$ \\
\hline Avaliação & É verificado o quanto os objetivos foram alcançados \\
\hline Término & $\begin{array}{l}\text { A propriedade do sistema é transferida para a área usuária. É estabelecida a forma de } \\
\text { acompanhamento periódico para rastrear problemas. }\end{array}$ \\
\hline Fonte: Baseado em Kolb e Frohman (1970) e Pinto e Millet (1999)
\end{tabular}

Quadro 1. Modelo de Kolb e Frohman aplicado à implementação de sistemas de informação

Quanto aos estudos sobre fatores, o modelo de fatores críticos de sucesso - FCS - na implementação de projetos (Quadro 2) (SCHULTZ, SLEVIN e PINTO, 1987) oferece um referencial para o gerenciamento do projeto de implementação de sistemas de informação. Do ponto de vista do estágio a que estão relacionados, é possível considerar que os fatores missão do projeto, suporte da alta gerência e plano do projeto estão associados ao estágio inicial e dizem respeito a atividades estratégicas do projeto (PINTO e MILLET, 1999). Por outro lado, os fatores consultoria ao cliente, seleção e treinamento de pessoal, tarefas técnicas, aceitação pelo cliente, feedback, comunicação e tratamento de problemas estão relacionados ao estágio de operacionalização e caracterizam-se como fatores de nível tático (PINTO e MILLET, 1999).

\begin{tabular}{|l|l|}
\hline FCS & Descrição \\
\hline Missão do projeto & $\begin{array}{l}\text { Definir objetivos do projeto de forma clara e de comum acordo entre os } \\
\text { participantes. }\end{array}$ \\
\hline Suporte da alta gerência & Obter o suporte da alta gerência em termos de disponibilização dos recursos. \\
\hline Plano do projeto & $\begin{array}{l}\text { Especificar detalhadamente os passos a serem seguidos no processo de } \\
\text { implementação, incluindo as especificações dos recursos exigidos. }\end{array}$ \\
\hline Consultoria ao cliente & $\begin{array}{l}\text { Realizar comunicação, consultoria e escuta das partes impactadas pelo projeto, para } \\
\text { especificar as necessidades a serem atendidas. }\end{array}$ \\
\hline Pessoal & Recrutar, selecionar e treinar equipe de implementação do projeto. \\
\hline Tarefas técnicas & $\begin{array}{l}\text { O projeto deve ser gerenciado por pessoas familiarizadas com ele e que possuam } \\
\text { habilidades e acesso à tecnologia necessária para realizar as tarefas. }\end{array}$ \\
\hline Aceitação pelo cliente & Proceder a venda do produto final para os usuários finais pretendidos. \\
\hline Monitoração e feedback & $\begin{array}{l}\text { Prover informação de controle para cada estágio no processo de implementação } \\
\text { com o objetivo de averiguar se as projeções iniciais estão sendo alcançadas. }\end{array}$ \\
\hline
\end{tabular}




\begin{tabular}{|l|l|}
\hline Comunicação & $\begin{array}{l}\text { Dispor de canais de comunicação formais e informais para disseminar informações } \\
\text { sobre o andamento do projeto e a troca de experiências. }\end{array}$ \\
\hline Tratamento de problemas & Capacidade de manusear crises não esperadas e desvios do que foi planejado. \\
\hline
\end{tabular}
Fonte: baseado em Schultz, Slevin e Pinto (1987)

Quadro 2: Definição dos fatores críticos de sucesso da implementação de projetos

A atuação sobre o fatores críticos de sucesso em cada estágio da implementação pode ser melhor detalhada a partir da integração do modelo de Schultz, Slevin e Pinto ao modelo de Kolb e Frohman. O modelo de Kolb e Frohman prescreve a relação entre o profissional de sistemas de informação e os usuários ao longo de etapas que detalham as atividades de cada estágio do modelo de Schultz, Slevin e Pinto (Figura 1).

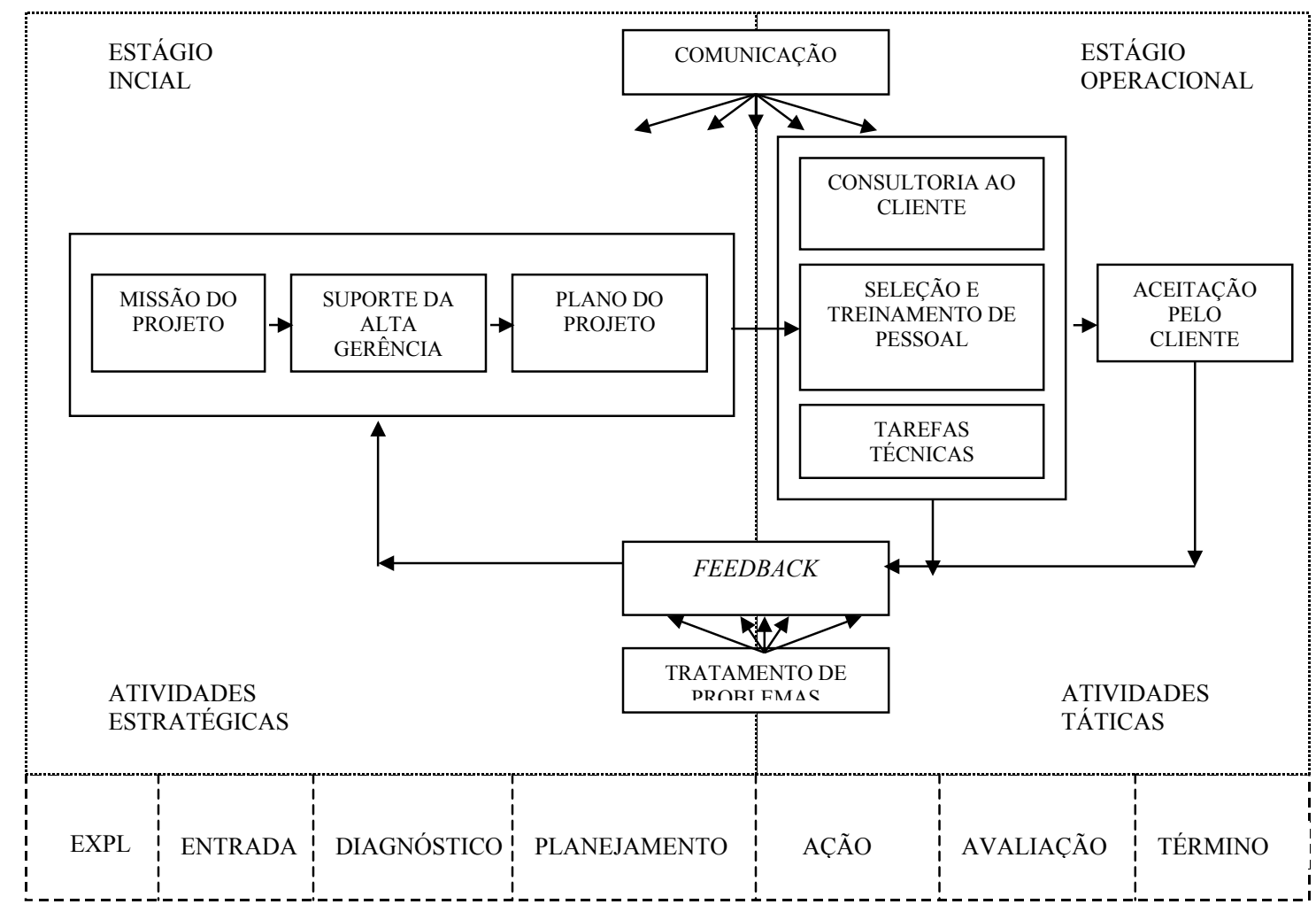

Figura 1: Integração entre o modelo de Schultz, Slevin e Pinto e o modelo de Kolb e Frohman

A partir da integração entre o modelo de Schultz, Slevin e Pinto e o modelo de Kolb e Frohman é possível discutir as competências para o gerenciamento do projeto de implementação de sistemas de informação.

\section{As competências para o gerenciamento da implementação de sistemas de informação}

O termo competência se refere ao conjunto de características que permite a execução de uma tarefa de forma a imprimir um nível de qualidade ao processo e ao produto que corresponda ou supere padrões de eficácia e eficiência estabelecidos. Deve-se considerar que uma competência é uma característica que está relacionada ao desempenho efetivo ou superior no trabalho e pode ser observada comportamentalmente (McCLELLAND, 1973). Isto permite sua descrição, análise, avaliação e desenvolvimento (McCLELLAND, 1973).

Apesar da diversidade de abordagens que se dá ao tema das competências, é possível considerar que os elementos de uma competência podem ser enquadrados em três eixos que dizem respeito às atitudes (saber ser), conhecimentos (saber) e habilidades (saber-fazer) 
necessários para o desempenho de uma atividade (RUAS, 2001) (Figura 2).

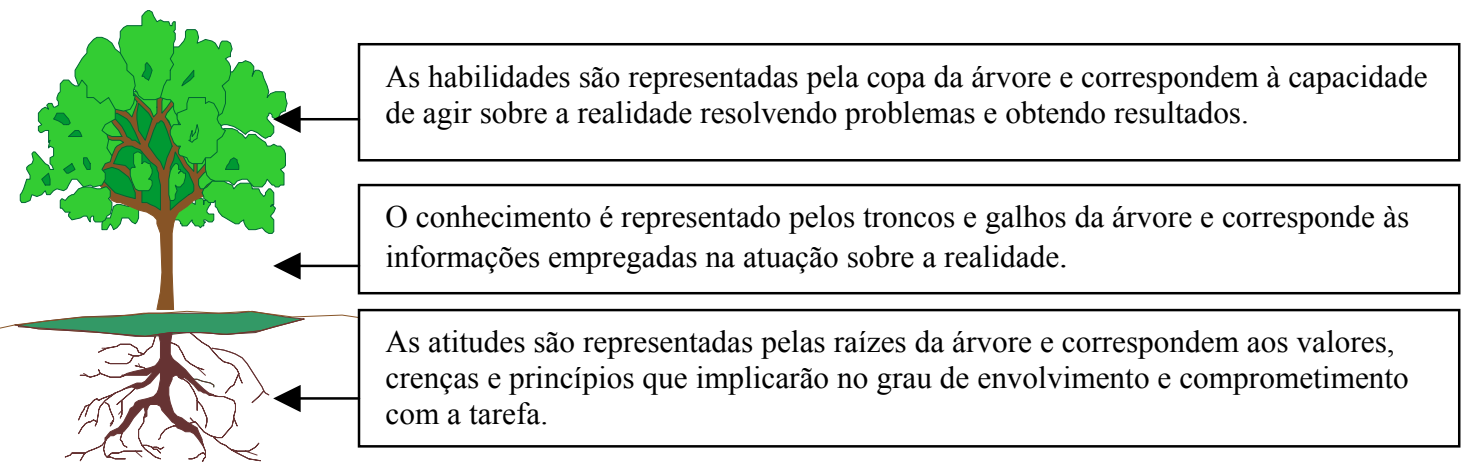

Figura 2. A árvore da competência

A especificação das competências para o gerenciamento da implementação de sistemas de informação deve considerar as funções dos membros da equipe e algumas características comuns a todo profissional que atua dentro da perspectiva do gerenciamento de projetos.

Com relação às funções desempenhadas pelos participantes de uma equipe de projeto, a proposta de Frame (1999) está baseada no corpo de conhecimento do gerenciamento de projetos do Project Management Institute - PMI - (PMI, 2000) (Quadro 3).

\begin{tabular}{|l|l|}
\hline Função & Descrição \\
\hline Gerente do projeto & É o responsável pelo gerenciamento do projeto. \\
\hline Patrocinador do projeto & É o responsável por criar o ambiente onde a equipe trabalhe efetivamente. \\
\hline Pessoal técnico & São os responsáveis pela produção dos resultados do projeto. \\
\hline Gerentes funcionais & São responsáveis pelo controle dos recursos empregados no projeto. \\
\hline Suporte & São responsáveis pelo suporte às atividades dos integrantes do projeto. \\
\hline
\end{tabular}

Quadro 3: Funções genéricas da equipe de projeto

De acordo com as responsabilidades atribuídas a uma determinada função, certas competências poderão ser mais importantes, entretanto há competências (Quadro 4) que fazem parte do perfil de todo profissional que atua em projetos (FRAME, 1999).

\begin{tabular}{|l|l|}
\hline Competência & Descrição \\
\hline $\begin{array}{l}\text { Competências } \\
\text { baseadas em } \\
\text { conhecimento }\end{array}$ & $\begin{array}{l}\text { Dizem respeito ao domínio das áreas de conhecimento do gerenciamento de projetos mapeadas } \\
\text { pelo e detalhadas no Project Management Body of Knowledge (PMI, 2000). As áreas são o } \\
\text { gerenciamento de escopo, gerenciamento de tempo, gerenciamento de custos, gerenciamento de } \\
\text { recursos humanos, gerenciamento de riscos, gerenciamento de qualidade, gerenciamento de } \\
\text { suprimentos, gerenciamento de comunicação e o gerenciamento de integração. }\end{array}$ \\
\hline $\begin{array}{l}\text { Competências } \\
\text { sociais }\end{array}$ & $\begin{array}{l}\text { Dizem respeito à capacidade de exercer um relacionamento interpessoal que facilite o trabalho } \\
\text { em equipe e o alcance dos objetivos. Incluem o trabalho em equipe, habilidades políticas, } \\
\text { habilidades de diversidade, comunicação e escuta. }\end{array}$ \\
\hline $\begin{array}{l}\text { Competências } \\
\text { de negócio }\end{array}$ & $\begin{array}{l}\text { Dizem respeito à capacidade de tomar decisões consistentes com os interesses da organização e } \\
\text { incluem sensibilidade de negócios e conhecimento de negócios. }\end{array}$ \\
\hline Fonte: baseado em Frame (1999)
\end{tabular}

Quadro 4. Tipologia de competências para o gerenciamento de projetos

As competências sociais merecem destaque pela dificuldade em sua avaliação e desenvolvimento e sua importância para a resolução de problemas humanos e organizacionais 
que têm sido relatados como os principais fatores que levam os projetos ao fracasso (FRAME, 1999). Na perspetiva de Frame (1999), embora os profissionais devam alcançar um balanceamento entre os três tipos de competências, é mais fácil capacitar e avaliar alguém nas competências baseadas em conhecimento e nas de negócio que nas competências sociais.

\section{A estrutura geral da metodologia de aprendizagem vivencial}

A estrutura geral da metodologia de aprendizagem vivencial (Figura 3) concebe o desenvolvimento das competências para o gerenciamento da implementação de sistemas de informação através de episódios. Cada episódio é uma simulação de atividades relacionadas aos estágios propostos pelo modelo de implementação de Kolb e Frohman. Em cada episódio os participantes enfrentarão um desafio que levará em conta a necessidade de atuação sobre determinados fatores críticos de sucesso, conforme o modelo de Schultz, Slevin e Pinto.

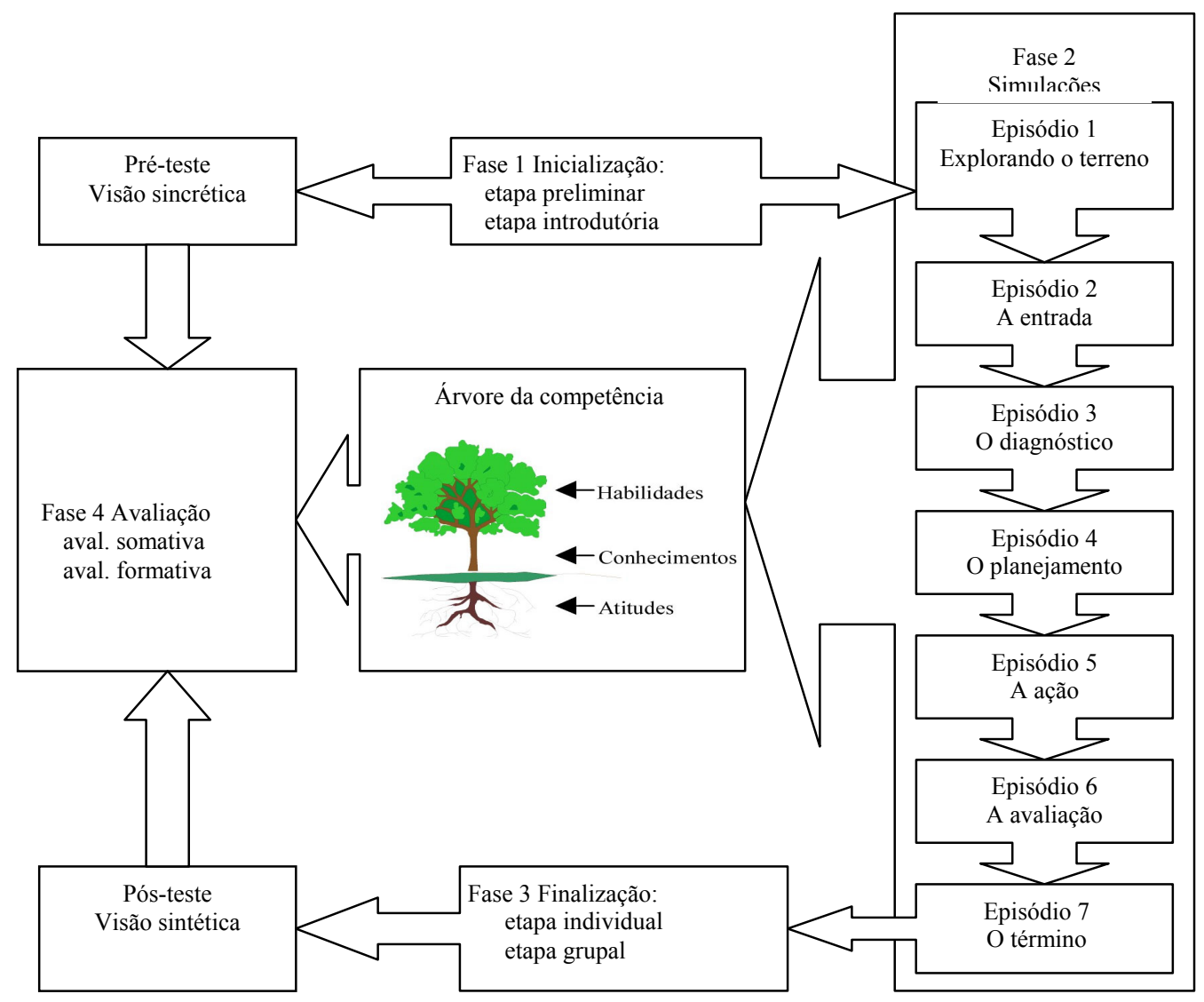

Figura 3. Estrutura geral da metodologia de aprendizagem

\subsection{Fase 1 - Inicialização}

Esta fase objetiva caracterizar o perfil dos participantes e apresentar as orientações iniciais a respeito das atividades a serem realizadas. $\mathrm{Na}$ etapa preliminar é aplicado um questionário individual para determinar o perfil acadêmico e profissional de cada participante, bem como o conhecimento e experiência prévios sobre implementação de sistemas de informação. $\mathrm{Na}$ etapa introdutória a metodologia é apresentada e as equipes formadas.

\subsection{Fase 2 - Simulações}

O objetivo desta fase é propiciar uma seqüência de episódios que simulam ocorrências ao longo do processo de implementação de um sistema de informação. Cada episódio é estruturado como um processo de aprendizagem vivencial que emprega a técnica de simulação. Ao longo dos episódios os participantes produzem relatórios sobre as discussões 
realizadas e constróem uma árvore composta pelas atitudes, conhecimentos e habilidades necessários para atuar na implementação de sistemas de informação.

\subsection{Fase 3 - Finalização}

Esta fase tem por meta caracterizar as sínteses individual e grupal do processo de aprendizagem. Na etapa individual é aplicado um questionário para delinear o conhecimento adquirido sobre a implementação de sistemas de informação e sobre as atitudes, conhecimentos e habilidades necessários para empreender este processo. Na etapa grupal é realizado um painel para sintetizar as conclusões das equipes.

\subsection{Fase 4 - Avaliação}

Nesta fase o objetivo é proceder as avaliações somativa e formativa da metodologia de aprendizagem. A avaliação somativa é a análise comparativa entre a visão sincrética e a visão sintética dos participantes e a discussão em grupo das árvores de atitudes, conhecimentos e habilidades construídas ao longo do processo. A avaliação formativa é análise dos dados coletados ao longo da aplicação da metodologia através de observações e dos relatórios produzidos pelos participantes em cada episódio.

\section{A estrutura geral dos episódios vivenciados}

Os episódios que compõem a Fase 2 segue o processo de aprendizagem vivencial (MOSCOVICI, 2000) apresentado na Figura 4.

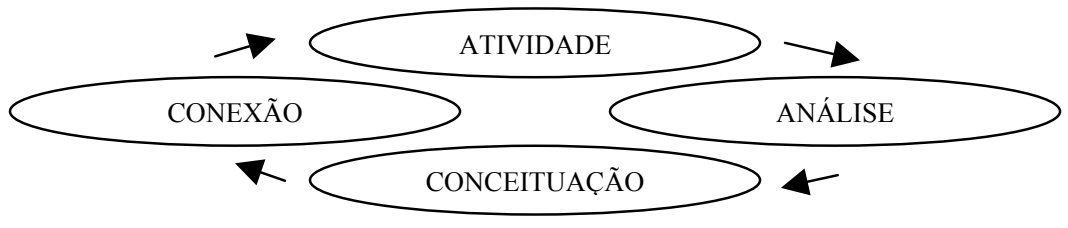

Figura 4: Estrutura geral dos episódios

\section{1. $1^{\mathrm{a}}$ etapa: atividade}

Através de uma simulação, os participantes deverão propor estratégias de solução para as questões que lhes sejam apresentadas e que dizem respeito a atuação em um determinado fator crítico de sucesso da implementação de projetos.

\section{2. $2^{\mathrm{a}}$ etapa: análise}

Através de um roteiro de discussão, cada equipe registra as decisões tomadas, tarefas realizadas e resultados obtidos no episódio.

\section{3. $3^{\text {a }}$ etapa: conceituação}

Através de um brainstorming e de um roteiro de mapeamento, cada equipe registra os aspectos que dificultaram as decisões e ações no episódio e as alternativas que poderiam ter sido buscadas. Além disso, cada equipe realiza o mapeamento das atitudes, conhecimentos e habilidades necessários para atuar sobre o fator crítico de sucesso daquele episódio.

\section{4. $4^{\text {a }}$ etapa: conexão}

Através da comparação dos aspectos abordados nas demais etapas com situações de trabalho, cada equipe elabora um relatório de conclusão contendo generalizações para uso futuro.

\section{A estrutura geral das simulações}

As simulações a serem realizadas em cada episódio da Fase 2 apresentam uma estrutura de 
acordo com a proposta de Gredler (1994):

- objetivo: identificar e desenvolver as competências para atuar sobre os fatores críticos de sucesso relacionados àquele episódio;

- estrutura: aberta, conforme conceituado por Gredler (1994);

- problema central: o problema está relacionado aos fatores críticos de sucesso associados àquele episódio específico, na forma de questões apresentadas aos participantes;

- papéis: os papéis a serem simulados incluem as funções genéricas de uma equipe de projeto, conforme abordado por Frame (1999);

- cena de abertura: é a descrição de um evento organizacional relacionado ao estágio de implementação a que o episódio se refere;

- estímulo: uma questão ou problema relacionado ao episódio em simulação e que sintetize as decisões centrais a serem tomadas naquele estágio da implementação;

- reações às decisões e ações realizadas pelos participantes: alterações no status do problema levando em conta a atuação sobre os fatores críticos de sucesso;

- atividades de avaliação e feedback: correspondem as etapas de análise, conceituação e conexão da estrutura geral do episódio (Figura 4).

\section{O papel do coordenador e dos observadores}

O coordenador desempenha as funções de arquiteto, consultor e facilitador do processo de aprendizagem. Como arquiteto é responsável por definir a estrutura geral da simulação. Como consultor atua na medida que os participantes demandem mais informações a respeito das situações vivenciadas.Como facilitador faz com que os participantes percebam os processos interpessoais e grupais e suas implicações para o trabalho em equipe.

Sobretudo nas situações onde o número de participantes é elevado, é possível dispor de observadores para auxiliar na coleta de dados durante a aplicação da metodologia de aprendizagem.

\section{A aplicação da metodologia}

Atualmente a metodologia vem sendo aplicada em turmas de graduação e pós-graduação na área de Computação e Informática e na área de Engenharia de Produção na UNIVILLE, UDESC e UFSC. As aplicações ocorrerão ao longo de sete semanas, sendo que a cada semana os alunos estarão simulando um episódio relativo a um estágio do modelo de implementação proposto na Figura 1. Adicionalmente será empregada uma oitava semana para a realização do fechamento das atividades através de um seminário onde, em cada turma, as diversas equipes estarão apresentando suas conclusões. A situação organizacional que está sendo simulada é a de uma empresa que conta com sistemas não integrados e pretende implementar um sistema integrado de gestão empresarial.

\section{Conclusão}

Embora os sistemas de informação sejam peça fundamental nas organizações, nem sempre a sua implementação tem alcançado o objetivo a que se propõe. Estatísticas mostram que cerca de $31 \%$ dos projetos de software são cancelados, $51 \%$ dos projetos que chegam ao fim custam duas a três vezes mais do que o orçado e levam três vezes mais tempo do que o previsto e $75 \%$ dos grandes sistemas operam com falhas (LAUDON e LAUDON, 1999). Boa parte destes problemas tem causas organizacionais e humanas. Estes dados justificam a necessidade de avançar no desenvolvimento das competências de gerenciamento de implementação de 
sistemas. Neste sentido, o presente artigo apresentou a fundamentação teórica e o delineamento de uma metodologia de aprendizagem vivencial que visa o desenvolvimento de competências de gerenciamento de projetos de implementação de sistemas de informação.

É importante destacar a necessidade crescente da busca de novos métodos de ensinoaprendizagem voltados para o desenvolvimento de competências cada vez mais necessárias na atuação profissional, como é o caso das relacionadas ao trabalho em equipe e ao gerenciamento de projetos. Assim, a aplicação da metodologia está em sua fase inicial e os resultados esperados dizem respeito à operacionalização de ações efetivas no desenvolvimento de atitudes, conhecimentos e habilidades aplicados ao gerenciamento da implementação de sistemas de informação.

\section{Referências}

APPLEGATE, L. M. et al. (1999) - Corporate information systems management. Irwin/McGraw-Hill. $5^{\text {a }}$ Edição. New York.

BIKSON, T. K. \& EVELAND, J. D. (1990) - Technology transfer as a framework for understanding social impacts of computerization. Rand Corporation. New York.

FRAME, J. D. (1999) - Project management competence. Jossey-Bass. San Francisco.

GINZBERG, M. J. (1979) - A study of the implementation process. TIMS Studies in the management sciences. Vol. 13, p. 85-102.

GREDLER, M. (1994) - Design and evaluating games and simulations: a process approach. Gulf Publishing Company Houston.

KOLB, D. A. \& FROHMAN, A. L. (1970) - An organization development approach to consulting. Sloan management review. Vol. 12, n. 1, p. 51-65, fall.

KWON, T. H. \& ZMUD, R. W. (1987) - Unifying the fragmented models of information systems implementation. In: BOLAND, R. J. \& HIRSCHHEIM, R. A. Critical issues in information systems research., John Wiley e Sons. New York. p. 227-252.

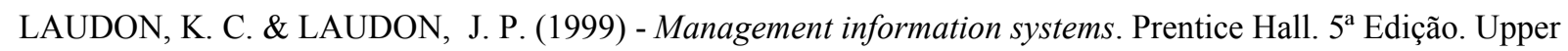
Saddle River.

LUCAS, H. C., GINZBERG, M. J. \& SCHULTZ, R. L. (1990) - Information systems implementation: testing a structural model. Ablex Publishing Corporation. New Jersey.

LUCAS, H. C. (1981) - Implementation: the key to successful information systems. Columbia University Press. New York.

LYYTINEN, K. \& HIRSCHHEIM, R. (1987) - Information systems failures: a survey and classification of the empirical literature. Oxford Surveys in information technology. Vol. 4, p. 257-309.

McCLELLAND, D. C. (1973) - Testing for competence rather than 'intelligence'. American Psychologist. Vol. 28 , n. 1 , p. $1-40$.

MOSCOVICI, F. (2000) - Desenvolvimento interpessoal: treinamento em grupo. José Olympio. $9^{\mathrm{a}}$ Edição. Rio de Janeiro.

PINTO, J. K. \& MILLET, I.(1999) - Successful information system implementation: the human side. PMI. $2^{\text {a }}$ Edição. Pennsylvania.

PMI. (2000) - PMBOK® guide: a guide to the project management body of knowledge. PMI, Pennsylvania.

RUAS, R. (2001) - Desenvolvimento de competências gerenciais e contribuição da aprendizagem organizacional. In: FLEURY, M. T., OLIVEIRA Jr., M. M. Gestão estratégica do conhecimento. Atlas. São Paulo.

SCHULTZ, R. L., SLEVIN, D. P. \& PINTO, J. K. (1987) - Strategic and tatics in a process model of project implementation. Interfaces. Vol. 17, n. 3, p. 34-46, maio-junho.

TORNATZKY , L. G. \& FLEISCHER, M. (1990) - The process of technological innovation. Lexington Books New York. 